\title{
56Gbps Signal Generation from one 10-G Class Laser Diode for 400G Intra-data Center Interconnection
}

\author{
Kaibo Fang ${ }^{1}$, Jianjun $\mathrm{Yu}^{1,2^{*}}$, Hada Wang ${ }^{1}$, and Xinying $\mathrm{Li}^{1,2}$ \\ 1)Key Laboratory for Information Science of Electromagnetic Waves (MoE), Fudan University, Shanghai 200433, China \\ 2) ZTE (TX) Inc., Morristown, NJ 07960,USA (jianjun@fudan.edu.cn),
}

\begin{abstract}
We have demonstratedup to 56-Gb/s cost-efficient transmission forintra-data center interconnection over $10-\mathrm{km}$ SMF-28with negative power penalty. This $56-\mathrm{Gb} / \mathrm{s}$ transmitter comprisesa low-cost 10-G-class directly-modulated distributed-feedback (DFB) laser without requiring expensive digital to analog converter (DAC), analog-to-digital converter (ADC) and power-consumptivedigital signal processing (DSP).
\end{abstract}

Keywords: directly-modulated distributed-feedback, interconnection, data center, optical fiber communication.

\section{Introduction}

With the popularization of data centre and other bandwidth hungry inter-connect applications, the desired capacity of short reach optical network has exponentially increased to 400Gbit/s or even more. Recent standardization efforts for $400 \mathrm{G}$ intra-data center connections specify link lengths of up to $2 \mathrm{~km} \mathrm{[1,2].8 \times 56} \mathrm{Gb/s} \mathrm{could} \mathrm{enable} \mathrm{such} 400-\mathrm{G}$ networks. Relative to coherent detection [3], intensity modulation/direct detection (IM/DD) is a good candidate in inter-connect due to its low cost [4-8].For 56-Gb/s signal generation, a few modulation formats or schemes, such as pulse-amplitude-modulation (PAM4)[3-5] anddiscretemulti-tone(DMT)[6-8], are proposed and experimentally demonstrated. However, these modulation formats need expensive DAC and ADC as well as DSP procession [3-8]. Here we propose and experimentally demonstrate to use 56Gb/s CML signal to transmit over 10km SMF-28 with one negative power penalty. This $56-\mathrm{Gb} / \mathrm{s}$ transmitter comprisesa $10-\mathrm{G}$ class directly modulated distributed-feedback (DFB) laserand a subsequent optical interleaver. Large dispersion tolerance of this transmitter is realized by chirp control through the phase correlation between adjacent bits for the destructive interference in order to erase the power of " 0 " bits while enhancing the extinction ratio (ER) [9-12]. In our system, we do not need any DAC, ADC or DSP, so it is one real low-cost solution for high-speed intra-data connection. After transmission over 10-km SMF-28 without any optical amplifier, there is 6.3-dB power margin.

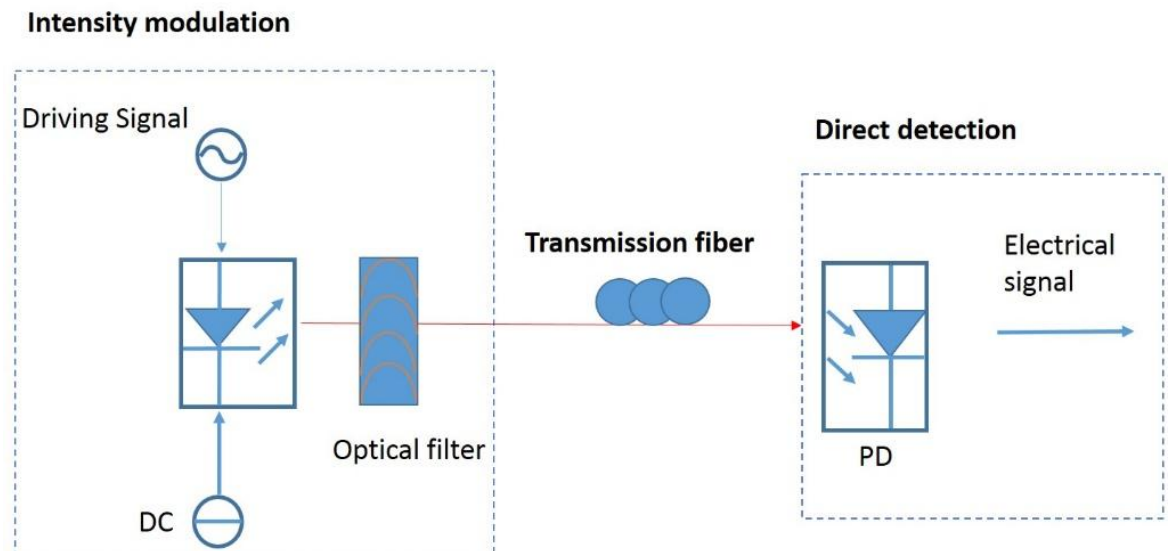

(a)

(C) 2016. This manuscript version is made available under the Elsevier user license 


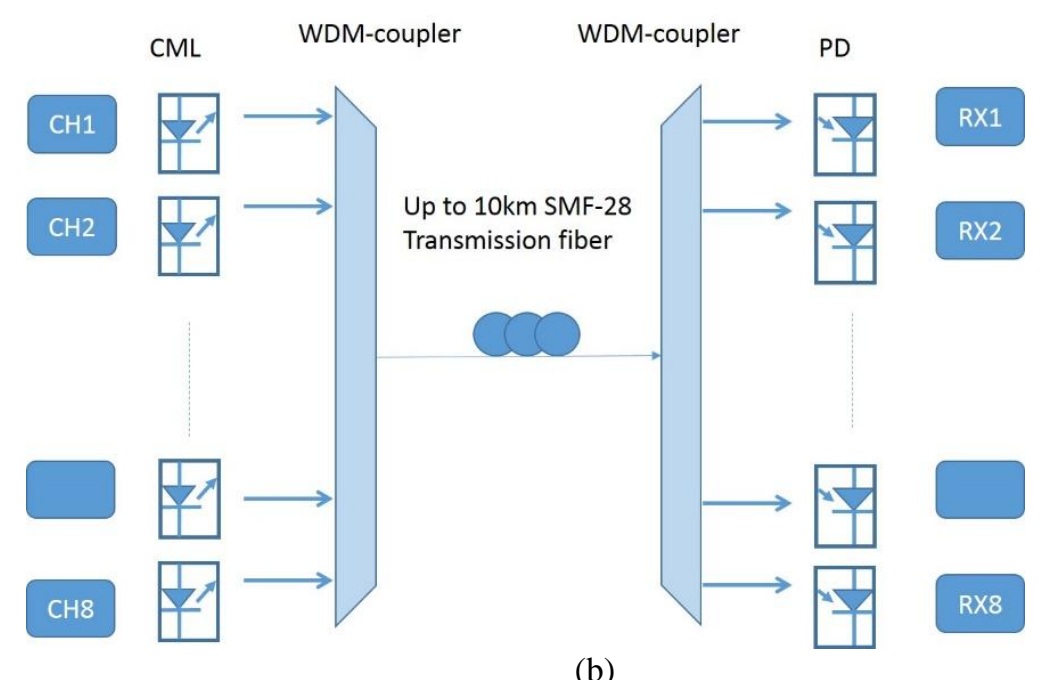

Fig. 1. (a)Schematic of chirp-managed DML transmitter. (b) Solution of 400G (8x56Gps CML signal) data interconnection.

\section{Principle}

The CML transmitter comprises a DML and the subsequent optical filter, and its schematic diagram is shown in Fig. 1(a) [10-13]. It is based on low cost and simple architecture with intensity modulation and direct detection. The laser is operated at high DC bias and RF driving voltage. One optical filter is used to manage the chirp and enhance the ER. This optical filter can be one regular optical bandpass filter, AWG, WDM coupler, optical interleaver or other optical filter [10-13]. To realize the proper phase flip between the bits, a much higher driven bias compared to the conventional direct modulation is employed. The additional benefits of the higher bias are the higher output power and wider modulation bandwidth due to the high operation point $[9-10,13]$. We can also achieve the stable signal mode operation and low timing jitter, and make the laser to be the adiabatic chirp dominated via suppression of the transient chirp because the working condition is far away from the threshold of the laser. Fig. 1(b) shows the solution of $400 \mathrm{G}$ optical interconnection based on $8 \times 56 \mathrm{G} \mathrm{CML}$. We can use one WDM-coupler or AWG to combine the eight CML signals operated at 56Gb/s. At the receiver we use another WDM-coupler or AWG to separate them before direct detection. We assume that the output power from DML is $10 \mathrm{dBm}$, WDM coupler has $1.5 \mathrm{~dB}$ insertion loss, optical fiber has one insertion loss of $0.2 \mathrm{~dB} / \mathrm{km}$, and fiber length is $10 \mathrm{~km}$. There is additional loss of $\sim 1 \mathrm{~dB}$ caused by the CML generation when the center wavelength of the signal is tuned away from the passing wavelength of the optical filter. In this case, the optical power after transmission over 10km and two WDM couplers is $10-1.5-2-1.5-1=4 \mathrm{dBm}$. If consider one WDM-PON system with two 8:1 optical couplers with one total of $18 \mathrm{~dB}$ loss, then, the optical power is only $-14 \mathrm{dBm}$. In our following experiment in Fig. 3(c), we can see that the required optical power is $-2 \mathrm{dBm}$. It means that we do not have enough power budgetbecause over $12 \mathrm{~dB}(14-2=12)$ power increase is needed. To solve this problem, we can use one Avalanche photodiode (APD) receiver, one PIN receiver integrated one semiconductor optical amplifier (SOA), or one EDFA in the transmitter side. One APD usually has over 15dB receiver sensitivity higher than one PIN, and one SOA or EDFA can easily provide over $15 \mathrm{~dB}$ gain [15].

There are a few schemes for $50 \mathrm{~Gb} / \mathrm{s}$ signal generation and used in the intra-data center interconnection. These schemes include PAM4, DMT, CML, and On-Off keying (OOK). Table 1 summarizes the system performance when we consider the cost, transmission distance, bandwidth requirement for optical and electrical devices and power consumption. If the scheme needs ADC or DAC, usually the power consumption will be high. For PAM4 signal generation, one two bit DAC or one electrical multiplexer is necessary at the transmitter side, and one ADC or multi-level electrical signal detection is required at the receiver side. For DMT signal, DAC is required at the transmitter side, and ADC is required at the receiver side. For $56 \mathrm{~Gb} / \mathrm{s}$ OOK signal, over $40 \mathrm{GHz}$ bandwidth of optical and electrical devices is required for high-quality OOK signal generation.

Table. Performance comparison for $50 \mathrm{~Gb} / \mathrm{s}$ signal generation and used the intra-data center interconnection.

\begin{tabular}{|l|l|l|l|l|}
\hline Scheme & $\begin{array}{l}\text { Bandwidth } \\
\text { requirement for O/E } \\
\text { devices }\end{array}$ & $\begin{array}{l}\text { Transmission } \\
\text { distance without } \\
\text { dispersion } \\
\text { compensation }\end{array}$ & Power consumption & Cost \\
\hline OOK [16] & $\begin{array}{l}\text { Larger than } 40 \mathrm{GHz} \\
\text { for OOK signal and }\end{array}$ & Less than 2km & low & high \\
\hline
\end{tabular}




\begin{tabular}{|l|l|l|l|l|}
\hline & $\begin{array}{l}\text { larger than 30GHz } \\
\text { for duo-binary [15] }\end{array}$ & & & \\
\hline DMT [6] & $\sim 18 \mathrm{GHz}$ & Less than $20 \mathrm{~km}$ & high & high \\
\hline PAM4 [4] & $\sim 20 \mathrm{GHz}$ & Less than $10 \mathrm{~km}$ & high & high \\
\hline CML $[9,11]$ & $\sim 15 \mathrm{GHz}$ & Less than $15 \mathrm{~km}$ & low & low \\
\hline
\end{tabular}

\section{Experimental setup}

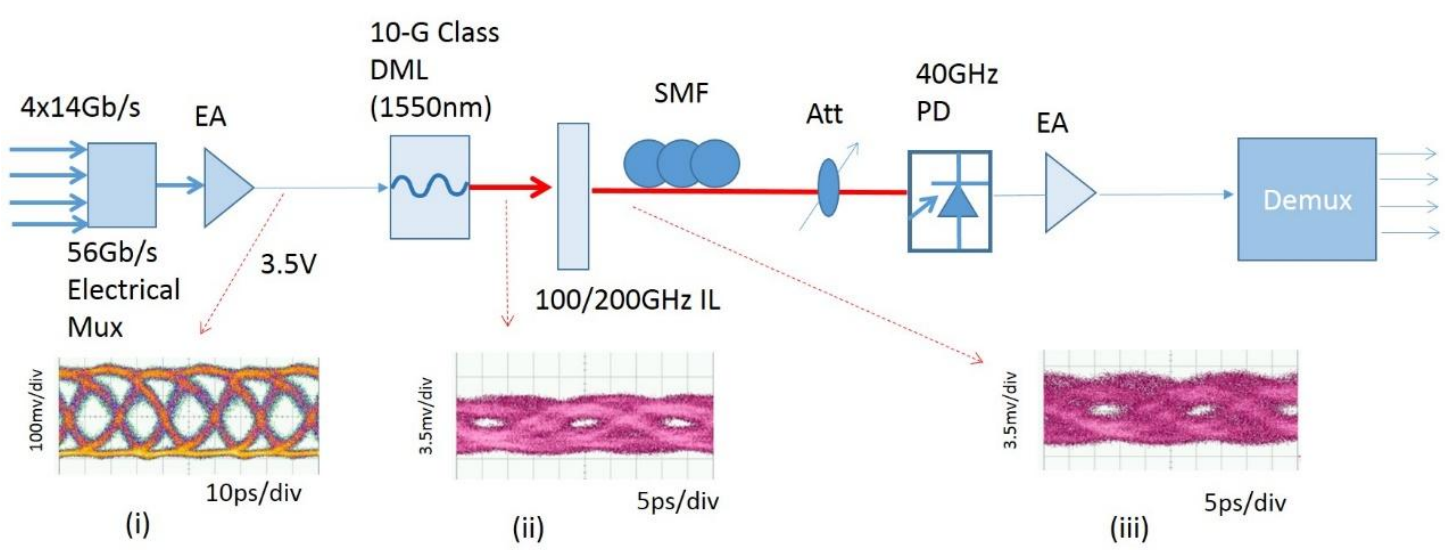

Fig. 2. Experimental setup of CML signalgeneration, transmission and direct detection.Red solid line: optical signal path. EA: electrical amplifier, PD: photodiode.

Fig. 2 shows the experimental setup. We use one electrical multiplexer to combine four channels $14 \mathrm{~Gb} / \mathrm{s}$ signal with different time delay for de-correlation purpose to generate $56 \mathrm{~Gb} / \mathrm{s}$ electrical signal. The $14 \mathrm{~Gb} / \mathrm{s}$ signal is generated from one PPG and the PRBS pattern length is $2^{31}-1$. Then we use one $40 \mathrm{GHz}$ RF amplifier to boost the signal to $3.5 \mathrm{~V}$ before it is used to drive one $10-\mathrm{G}$ class $1.55 \mu \mathrm{m}$ DML (NEL model number: NLK1551SSL). The electrical eye diagram after this RF amplifier is inserted in Fig.2 as inset (i). This DML is DC biased at 130mA with one output power of $10 \mathrm{dBm}$.The DC bias for the operated linearregion of this laser isfrom 20 45mA. Obviously this laser operates at the nonlinear area, and it has one large output power of $10 \mathrm{dBm}$ and wide modulation bandwidth. The eye diagram before the optical interleaver is inserted in Fig. 2 as inset (ii). The measured ER is $2 \mathrm{~dB}$. We use one $100 / 200 \mathrm{GHz}$ optical interleaver to generate CML signal. This $100 / 200 \mathrm{GHz}$ interleaver has one $3 \mathrm{~dB}$ passband of $100 \mathrm{GHz}$ and $1.2 \mathrm{~dB}$ insertion loss. The eye diagram after passing through this optical interleaver is inserted in Fig. 2 as inset (iii) with one measured ER of $3.8 \mathrm{~dB}$. Insets (ii) an (iii) are measured by using one $65 \mathrm{GHz}$ optical port of the sampling oscilloscope. We measured BER performance after this $56 \mathrm{~Gb} / \mathrm{s}$ signal passing through 4.4 20km SMF-28 with one insertion loss of $0.2 \mathrm{~dB} / \mathrm{km}$ and GVD of $17 \mathrm{ps} / \mathrm{nm} / \mathrm{km}$. At the receiver, one $40 \mathrm{GHz}$ optical receiver and a subsequent $40 \mathrm{GHz}$ low noise amplifier is used to realize $\mathrm{O} / \mathrm{E}$ conversion and amplification. We use one electrical de-multiplexer (Centellax MD1S4M) to de-multiplex the signal into four sub-channels with $1 / 4$ bit rate before BER test (Agilent 70843C). All BER of the four sub-channels are measured, and they show identical BER performance. The threshold of the electrical de-multiplexer is fixed in the middle value when the BER is measured. One optical attenuator is added before optical receiver for optical power change. There is no dispersion compensation or optical amplifier employed.

\section{Experimental results}




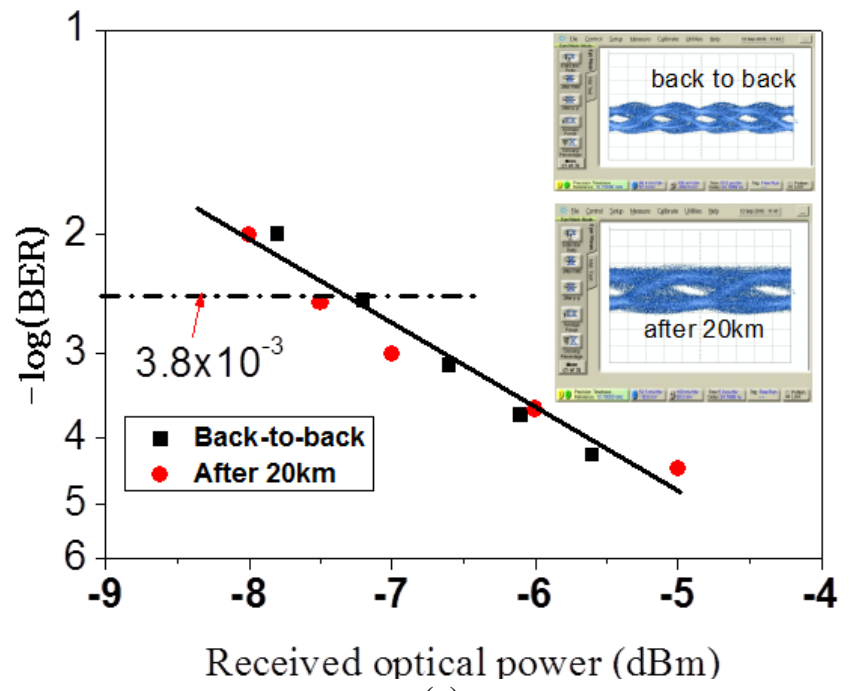

(a)

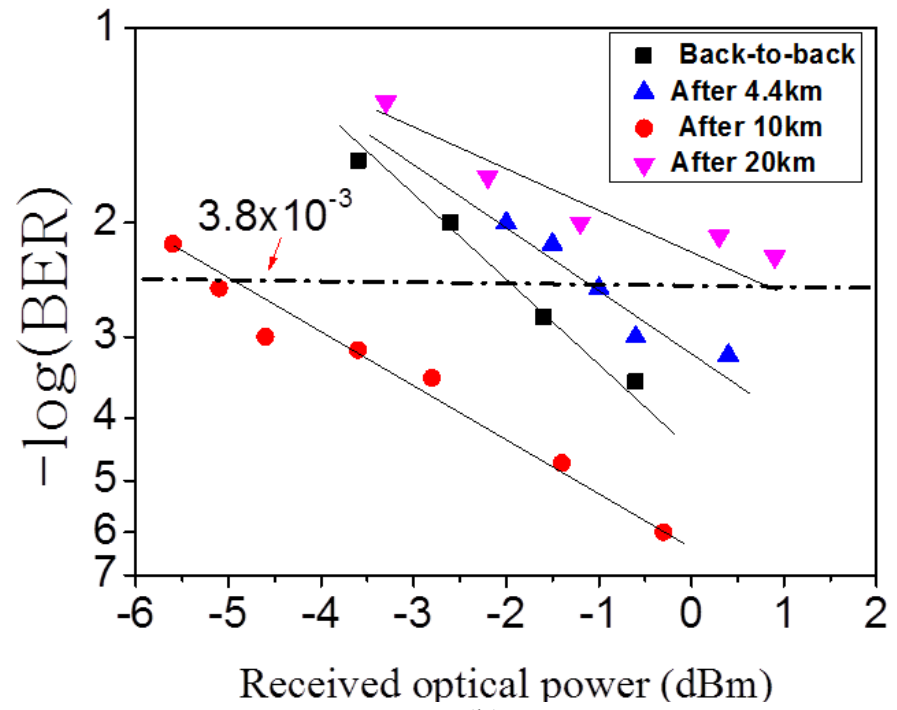

(b) 


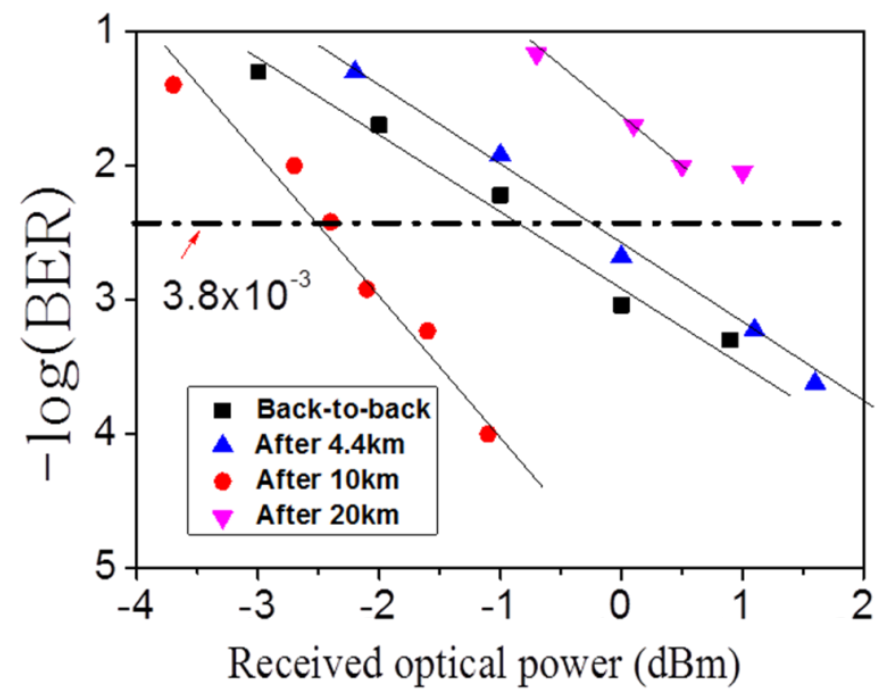

(c)

Fig. 3. (a) BER curves of $42.8 \mathrm{~Gb} / \mathrm{s}$ signal before and after $20 \mathrm{~km}$ SMF- 28 transmission. (b) BER curves of $50 \mathrm{~Gb} / \mathrm{s}$ signal.(c) BER curves of $56 \mathrm{~Gb} / \mathrm{s}$ signal.

We evaluated the BER performance of this CML operated at bit rates from $42.8 \sim 56 \mathrm{~Gb} / \mathrm{s}$ with different transmission distance. Fig. 3(a) shows the results at $42.8 \mathrm{~Gb} / \mathrm{s}$. We can see that there is no power penalty before and after transmission over 20km SMF-28. The measured eye diagrams after detected by the 40G PD before and after $20 \mathrm{~km}$ SMF-28 are inserted in this figure. The eye is clearly opened. The required optical power is $-7.6 \mathrm{dBm}$ to get BER of $3.8 \times 10^{-3}$. Fig. 3(b) shows the BER curves when the signal is operated at 50Gb/s. Due to fiber dispersion to change pulse shape of the CML signal, we can see that there is one quite large negative power penalty after transmission over $10 \mathrm{~km}$ SMF-28. But after transmission over $20 \mathrm{~km}$, the BER will be error-floor at 3x10 ${ }^{-3}$. Fig. 3(c) shows the BER curves at $56 \mathrm{~Gb} / \mathrm{s}$. After transmission over $10 \mathrm{~km}$, the power penalty is $-2 \mathrm{~dB}$ at FEC threshold of $3.8 \times 10^{-3}$. But it gets error-floor at $1 \times 10^{-2}$ after transmission over $20 \mathrm{~km}$. After transmission over $10 \mathrm{~km}$, we measured the optical power is $4 \mathrm{dBm}$, and it means that we have $6.3 \mathrm{~dB}(4-(-2.3)=6.3)$ power margin after transmission over $10 \mathrm{~km}$ SMF-28.

The measured P-I curve of the DML is shown in Fig. 4(a). The opticalspectraat 56Gb/s before and after optical interleaverareshown in Fig. 4(b). The optical transfer function of optical interleaver is also shown in this figure by blue line. The DML wavelength is $1532.8 \mathrm{~nm}$. We use one edge of the optical interleaver to realize the CML signal generation. Because of the periodical response of the optical interleaver, one optical interleaver can be used to realize multi-channel CML generation at different wavelength. Another advantage of optical interleaver is small insertion loss ofonly 1.2dB. Fig. 5 shows the eye diagrams after transmission over 4.4, 10 and $20 \mathrm{~km} \mathrm{SMF-28.}$

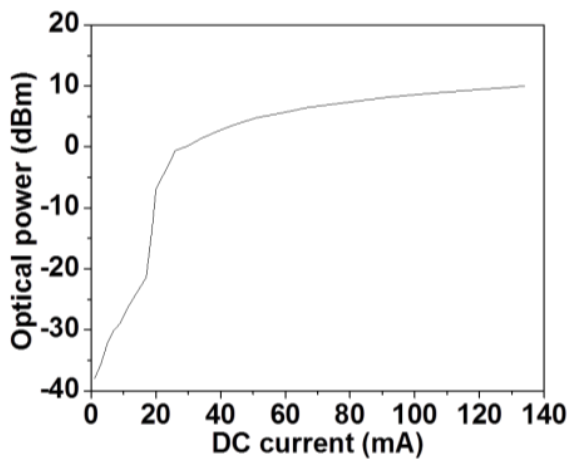

(a)

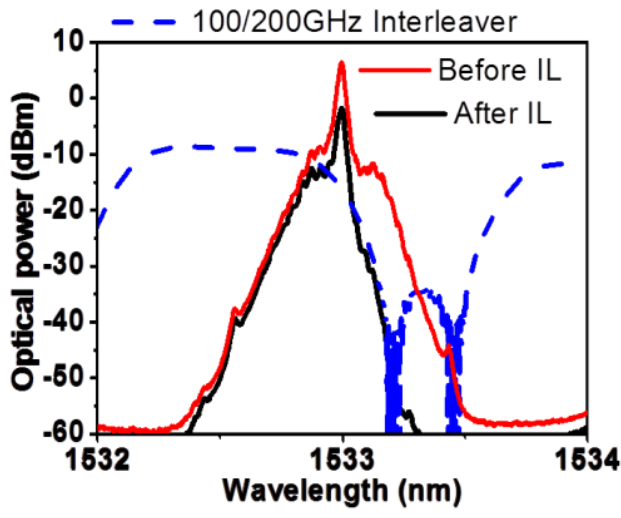

(b)

Fig. 4.(a) P-I curve of the DML, and (b)optical spectrabefore and after optical interleaver at 56Gb/s. 


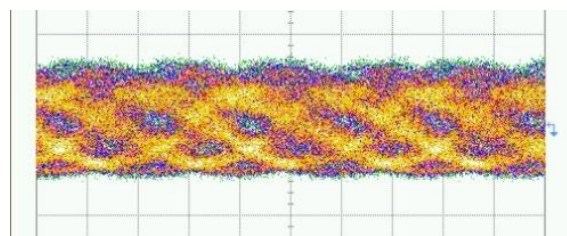

(a)

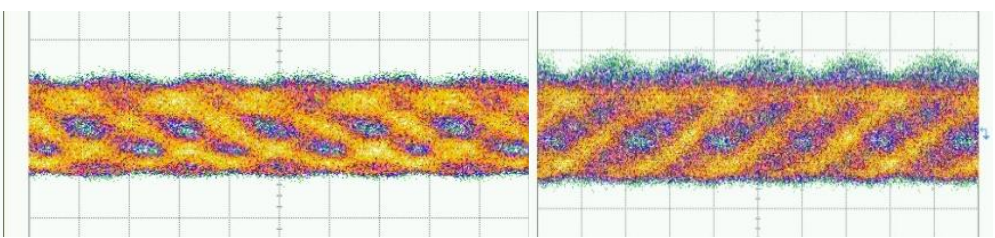

(b) (c)

Fig. 5.Eye diagrams (10ps/div)after(a) 4.4, (b) 10, and (c) $20 \mathrm{~km}$.

\section{Conclusion}

We have successfully transmitted56Gb/s CML signal by using one $10-\mathrm{G}$ class $1532.8 \mathrm{~nm}$ DML and a subsequent optical interleaver. After transmission over 10km SMF-28 without any dispersion compensation, there is $-2 \mathrm{~dB}$ power penalty and $6.3 \mathrm{~dB}$ power margin without any optical amplifier. In our demonstration, no expensiveADC, DAC and application-specific integrated circuit(ASIC) DSP chips are needed, whichrealizes low-cost operation for future $400 \mathrm{G}(8 \times 56)$ intra-data centerinterconnection.

\section{Acknowledgment}

This work was partly supported by China NSF with grant number of 61325002 and 61527801 . Authors would like to thank Dr. J. Zhang, Dr. H. Chien, and Prof. G. K. Chang for their support and help for this experiment.

\section{References}

1. Cisco Global Cloud Index. 2014-2019, white paper.

2. IEEE $802.3 \mathrm{bs} 400 \mathrm{~Gb} / \mathrm{s}$ Ethernet task force.

3. C. Xie, et al., "Generation and transmission of 100-Gb/s PDM 4-PAM using directly modulated VCSELs and coherent detection," OFC 2014, Paper Th3K2.

4. C. Chen, et al. "Transmission of 56-Gb/s PAM-4 over 26-km single mode fiber using maximum likelihood sequence estimation," OFC 2015, paper Th4A.5

5. F.Karinou, et al., " $56 \mathrm{~Gb} / \mathrm{s} 20-\mathrm{km}$ transmission of PAM-4 signal employing an EML in C-band without in-line chromatic dispersion compensation," ECOC 2016, paper W.4.P1.SC5.5.

6. A.Dochhan, et al., " $56 \mathrm{~Gb} / \mathrm{s}$ DMT transmission with VCSELs in 1.5 um wavelength range over up to $12 \mathrm{~km}$ for DWDM intra-data center connects," ECOC 2016, paper Th.1.C.1.

7. F. Li, et al., "Demonstration of four-channel CWDM 560 Gbit/s 128QAM-OFDM for optical inter-connection," OFC 2016, paper W4J.2.

8. A.Dochhan, et al.," $56 \mathrm{~Gb} / \mathrm{s}$ DMT Transmission with VCSELs in $1.5 \mathrm{um}$ Wavelength Range over up to $12 \mathrm{~km}$ for DWDM Intra-Data Center Connects", ECOC 2016, Tu.3.C.4.

9. Y. Matsui, et al., "Chirp-managed directly modulated laser(CML),"IEEE Photon. Technol. Lett. 18, 385-387 (2006).

10. J. Yu, M. F. Huang, P. N. Ji, and T. Wang., " $42.8 \mathrm{~Gb} / \mathrm{s}$ chirp-managedsignal transmission over $100 \mathrm{~m}$ graded-index plastic optical fiber,"OFC 2008, paper PDP28.

11. J Yu, Z Jia, MF Huang, M Haris, PN Ji, T Wang, GK Chang, "Applications of 40-Gb/s chirp-managed laser in access and metro networks, Journal of Lightwave Technology 27 (3), 253-265(2009).

12. J. Yu, J.Zhang,H. C.Chien, et al., "56Gb/s Chirp-managed Symbol Transmission with Low-cost, 10-G Class LD for 400G Intra-data Center Interconnection", OFC2017, W4D.2.

13. B. Dagens, A. Martinez, D. Make, O. L. Gouezigou, J. G. Provost, V. Sallet, K. Merghem, J. C. Harmand, A. Ramdane, and B. Thedrez, "Floor free 10-Gb/s transmission with directly modulated GaInNAs-GaAs 1.35-um laser for metropolitan applications," IEEE Photon. Technol. Lett., vol. 17, pp. 971-973, 2005.

14. J. Yu, P. N. Ji, Z. Jia, T. Wang, X. Zheng, Y. Matsui, D. Mahgerefteh, K. McCallion, Z. F. Fan, and P. Tayebati, “42.8 Gbit/s chirp-managed signal transmission over $20 \mathrm{~km}$ standard SMF at $1550 \mathrm{~nm}$ without DCF," Electron. Lett., vol. 43, 2007.

15. L.Yi, H. Ji, Z. Li, X. Li, et al., "Field -trial of a real -time $100 \mathrm{~Gb} / \mathrm{s}$ TWDM -PON based on 10G -class optical devices," Proc. ECOC, W 3.E.1, (2016)

16. S. H. Bae, Hoon Kim, and Y. C. Chung, "Transmission of $51.56-\mathrm{Gb} / \mathrm{s}$ OOK signal using $1.55-\mu \mathrm{m}$ directly modulated laser and duobinary electrical equalizer," Optics Express, 24, 22555-22562 (2016). 